\title{
Targeting mTORC1/2 with OSI-027 inhibits proliferation and migration of keloid keratinocytes
}

\author{
Jun Chen ${ }^{1, *}$, Ke Liu ${ }^{1, *}$, Yang Liu ${ }^{1}$, Xue Wang ${ }^{1}$ and Zhen Zhang ${ }^{1}$ \\ ${ }^{1}$ Department of Dermatology and Dermatologic Surgery, Shanghai Ninth People's Hospital, Affiliated to Shanghai Jiaotong \\ University School of Medicine, Center for Specialty Strategy Research of Shanghai JiaoTong University China Hospital \\ Development Institute, Shanghai 200011, China \\ *These authors contributed equally to this work
}

Correspondence to: Zhen Zhang, email: zz6503@126.com

Keywords: keloid keratinocytes; cell migration; mTOR; OSI-027; signaling

Received: August 08, $2017 \quad$ Accepted: December 11,2017 Published: January 02, 2018

Copyright: Chen et al. This is an open-access article distributed under the terms of the Creative Commons Attribution License 3.0 (CC BY 3.0), which permits unrestricted use, distribution, and reproduction in any medium, provided the original author and source are credited.

\section{ABSTRACT}

Keloid is a dermal proliferative disorder characterized by the excessive keratinocyte proliferation and migration. mTOR over-activation is involved in the process. Here, we show that both mTOR complex 1 (mTORC1) and mTORC2 were hyper-activated in keloid-derived primary keratinocytes. OSI-027, an mTOR kinase inhibitor, potently inhibited proliferation and migration of keloid keratinocytes. OSI027 disrupted the assembly of mTORC1 (mTOR-Raptor) and mTORC2 (mTOR-RictormLST8). Further, OSI-027 completely blocked the phosphorylations of the mTORC1 substrates (S6K1, S6 and 4EBP1) and the mTORC2 substrate (AKT, at Ser-473). OSI-027 was potent than rapamycin in inhibiting keloid keratinocytes. Moreover, restoring mTORC1 activation by the introduction of the constitutively active S6K1 only partly alleviated OSI-027-induced suppression on keloid keratinocytes. Notably, mTOR2 inhibition by Rictor siRNA also inhibited keloid keratinocyte proliferation and migration, although less efficiently than OSI-027. Together, concurrent targeting of mTORC1/ 2 by OSI-027 potently inhibits keloid keratinocyte proliferation and migration.

\section{INTRODUCTION}

Keloid is characterized by the formation of exuberant and autonomous scar tissue that extends the clinical border of the original wound [1-3]. It often forms as a result of dermal injuries, and is considered, to a limited extent, as a benign tumor [1-3]. A set of different chemokines, cytokines and growth factors are oversecreted, which stimulate the proliferation and migration of keratinocytes (and other skin cells) to form keloid skin [1-3]. The hyper-activation of the serine/threonine protein kinase mammalian target of rapamycin (mTOR) plays a pivotal role in the process [4-8].

Signaling by mTOR promotes cell proliferation and migration in a positive manner $[9,10]$. The active form of mTOR (phospho-mTOR) is over-expressed in keloid $[6,7]$. At least two multiple-protein mTOR complexes exist, including the traditional mTOR complex 1
(mTORC1), and the more recently discovered mTOR complex 2 (mTORC2) [9, 10]. Composed of mTOR, Raptor and PRAS40, mTORC1 is rapamycin sensitive $[11,12]$ and phosphorylates its target proteins, including p70S6K1 (S6K1) and eIF4E-binding protein 1 (4EBP1), to promote cell proliferation and migration [11-13]. The complex of mTORC2 primarily consists of mTOR, Rictor, Sin1 and mLST8 $[14,15]$ and functions as an AKT kinase, which phosphorylates AKT at Ser-473 [14, 15]. This mTORC2complex is also important for cell proliferation and migration $[16,17]$.

Recent studies have characterized OSI-027 as a novel and potent mTOR kinase inhibitor that displays activity against both mTORC1 and mTORC2 [18-20]. In the current study, we found that targeting mTORC1/2 withOSI-027 inhibited the proliferation and migration of keloid keratinocytes, where mTOR was found to be overactivated. 


\section{RESULTS}

\section{Enhanced cell proliferation and migration in keloid keratinocytes}

As described, primary human keratinocytes derived from keloid skin and surrounding normal skin were established and cultured. Viable cell counting assay results demonstrated that the keloid keratinocytes grew faster than the normal keratinocytes (Figure 1A). Meanwhile, an MTT OD assay further showed increased proliferation of keloid keratinocytes compared to normal keratinocytes (Figure 1B). Moreover, in keloid keratinocytes, BrdU (Figure 1C) and $\left[\mathrm{H}^{3}\right]$ DNA incorporation (Figure 1D) were both increased. These results demonstrate the increased cell proliferation of keloid primary keratinocytes compared to normal keratinocytes (Figure 1A-1D).

Migration of the above-mentioned cells was also tested using phagokinetic track motility $[4,21]$ and transwell assays. The former measured the migration distance of each single keratinocyte $[4,21]$, while the latter analyzed the number of migrated cells. As compared to normal keratinocytes, both migration distance (Figure 1E, quantified results are shown in the right panel) and the number of migrated cells (Figure 1F) were increased for keloid keratinocytes. Collectively, these results demonstrate enhanced cell proliferation and migration in keloid keratinocytes.

\section{OSI-027 potently inhibits proliferation and migration of keloid keratinocytes}

Figure 1 demonstrates that keloid keratinocytes showed increased cell proliferation and migration. We next analyzed mTOR signaling in keratinocytes. As shown in Figure 2A, phosphorylation ("p-") of mTORC1 substrates, including S6K1 and 4EBP1 [9, 10, 13], were significantly increased in keloid keratinocytes (compared to normal keratinocytes). Meanwhile, p-AKT (at Ser-473), an indicator of mTORC2 activation [14, 15], was also higher in keloid keratinocytes (Figure 2A). These results highlighted he over-activation of $\mathrm{mTORC} 1$ and mTORC2 in keloid keratinocytes (Figure 2A). Notably, the expression of total mTOR substrates, including S6K1, 4EBP1 and AKT1, were similar in keloid and normal keratinocytes (Figure 2A).

OSI-027 is a recently developed mTOR kinase blocker or dual mTORC1/2 inhibitor [18, 20]. Figure 2B-2D demonstrates that treatment with OSI-027 (100 nM, $72 \mathrm{~h})$ significantly attenuated proliferation of keloid keratinocytes, as determined by MTT (Figure 2B), BrdU ELISA (Figure $2 \mathrm{C}$ ) and $\left[\mathrm{H}^{3}\right]$ DNA incorporation assays (Figure 2D). Furthermore, the migration of keloid keratinocytes, tested again by phagokinetic track motility (Figure 2E; data are quantified in the right panel) and transwell assays (Figure 2F), was also suppressedbyOSI-027 (100 nM, 48 h). Intriguingly,
OSI-027 treatment ( $100 \mathrm{nM}, 72 \mathrm{~h}$ ) failed to induce the cell death of keloid keratinocytes as tested by propidium iodide (PI) staining assay (Figure 2G). As a positive control, $\mathrm{H}_{2} \mathrm{O}_{2}(300 \mu \mathrm{M})$ induced significant cell death (Figure $2 \mathrm{G}$ ). Collectively, these results confirm that OSI-027 inhibits proliferation and migration of keloid keratinocytes, where mTOR is over-activated.

\section{OSI-027 blocks mTORC1 and mTORC2 activation in keloid keratinocytes}

OSI-027 is a mTOR kinase inhibitor. The activation of mTOR in OSI-027treated keratinocytes was thus tested. Figure $3 \mathrm{~A}$ demonstrate that treatment with OSI-027 (100 nM, $2 \mathrm{~h})$ disrupted the assembly of both mTORC1 (mTOR-Raptor complex [22]) and mTORC2 (mTOR-Rictor-mLST8 complex [14, 23]) in keloid keratinocytes as determined by Co-IP. In contrast, the expression of mTOR complex proteins, including mTOR, Raptor, Rictor, and mLST8, was unchanged by OSI-027 (Figure 3A, Inputs). Further studies show that OSI-027 $(100 \mathrm{nM}, 2 \mathrm{~h})$ treatment almost completely blocked the phosphorylation ("p-") of mTORC1 substrates, including p70S6K1 (Thr-389), S6 (Ser-235/236) and 4EBP1 (Ser-65) [22] (Figure 3B), as well as that of the mTORC2 substrate, p-AKT (at Ser-473) [14, 23] (Figure 3C). The expression of total p70S6K1, S6, 4EBP1 and AKT1was unchanged, before and afterOSI-027 treatment (Figure 3B and 3C). Notably, ERK activation, detected by anti-p-ERK1/2, was unchanged after OSI-027 treatment (Figure 3D). These results suggest that OSI-027 simultaneously blocks mTORC1 and mTORC2 activation in keloid keratinocytes.

\section{mTORC1 and mTORC2 are both important in proliferation and migration of keloid keratinocytes}

Next, constitutivelyactiveS6K1 (T389E, "ca-S6K1flag-puro") [24] was introduced to keloid keratinocytes. As shown in Figure 4A, the introduction of ca-S6K1 completely restored S6K1 phosphorylation in OSI027treated keratinocytes.S6K1 phosphorylation was even higher than the control level (Figure 4A, quantification). The activation of mTORC2, detected by anti-p-AKT at Ser-473, was still blocked after OSI-027 treatment in ca-S6K1-expressing cells (Figure 4A). Intriguingly, inhibition of the OSI-027-induced proliferation (BrdU ELISA assay, Figure 4B) of keloid keratinocytes was only partly inhibited by ca-S6K1. Similarly, Figure 4C demonstrates that ca-S6K1 only partly restored the migration potentialofOSI-027-treated cells as determined by a phagokinetic track migration assay. It should be noted that the mTORC1 blocker, rapamycin (also at $100 \mathrm{nM}$ ) was less potent than OSI-027 in inhibiting the proliferation and migration of keloid keratinocytes (Figure 4B and 4C). These results imply that the inhibition of mTORC1 less 
potently inhibited keloid keratinocytes than OSI-027, suggesting that activation of both $\mathrm{mTORC} 1$ and $\mathrm{mTORC} 2$ is required in the process.

To further confirm our hypothesis, an siRNA strategy was applied to silence Rictor, which is the key component of mTORC2 [15]. In keloid keratinocytes, the two non-overlapping Rictor siRNAs ("Rictor siRNA I/II") both induced dramatic knockdown of Rictor (Figure 4D). Consequently, phosphorylation of the mTORC2 substrate, AKT (at Ser-473), but not the mTORC1 substrate, S6K1, was largely inhibited (Figure 4D). Notably, the silencing of Rictor by targeted siRNAs also inhibited proliferation (Figure 4E) and migration (Figure 4F) of keloid keratinocytes. However, Rictor siRNA-mediated inhibition of keloid keratinocytes was less potent than OSI-027 (Figure 4B-4F). These results further support the notion that mTORC1 and mTORC2 are both important for the proliferation and migration of keloid keratinocytes.

\section{DISCUSSION}

A skin keloid is characterized by the hyper-activity of keratinocytes (and other skin cells) within the wound [1-3].
Recent studies have confirmed mTOR hyperactivation as a potential therapeutic target for keloid $[5,7,8]$. It has been shown that the active form of mTOR (phospho-mTOR) is overexpressed in keloid but not in normal skin $[5,7,8]$. Rapamycin and other mTOR inhibitors were shown to efficiently inhibit keloid keratinocytes [5-7].

Rapamycin binds to FK506 binding protein-12 (FKBP12), to form the FKBP12-rapamycin complex that only partially inhibits mTOR kinase activity [25]. There are several limitations when using rapamycin and its analogs (rapalogs) [26-28]: First, rapalogs indirectly inhibit mTOR kinase activity (via binding to FKBP12), causing the inadequate inhibition of mTORC1 [26]. Second, mTORC1 inhibition induces feedback activation of several key mitogenic pathways, including AKT $[29,30]$ and Erk-MAPK [31]. Third, and most importantly, mTORC1 inhibitors showed no direct effect on MTORC2 activation, the latter promoting cell proliferation and migration $[16,17,32]$. Finally, the bioavailability of rapalogs is often limited [33].

Due to these limitations, direct mTOR kinase inhibitors have recently been developed, termed "secondgeneration mTOR inhibitors" [26]. OSI-027 is a novel

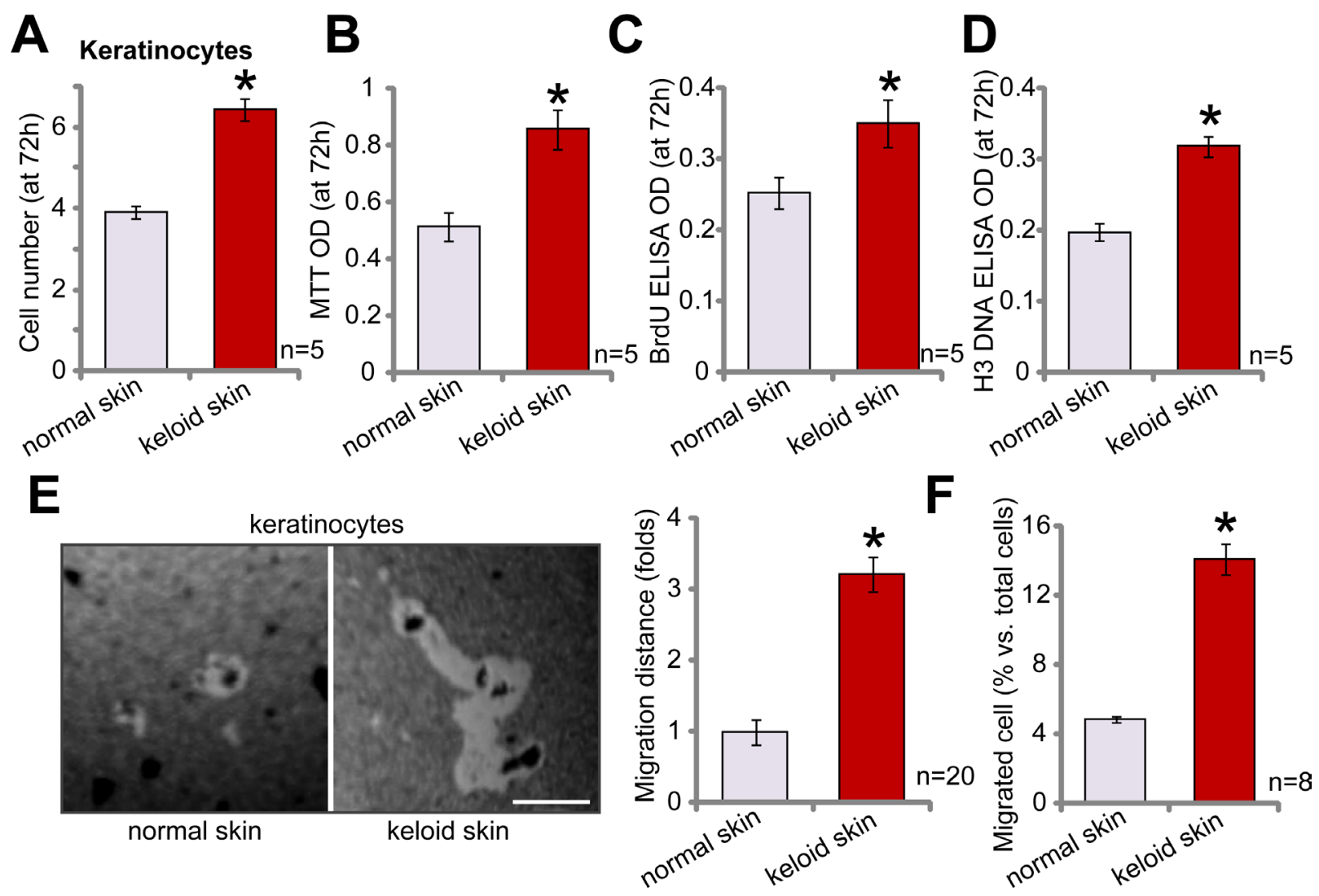

Figure 1: Enhanced cell proliferation and migration in keloid keratinocytes. Primary cultured human keratinocytes, derived from keloid and surrounding normal skin, were subjected to cell counting (A), MTT (B), BrdU ELISA (C) and [H ${ }^{3}$ ] DNA incorporation assays (D) to determine cell proliferation. Cell migration was analyzed using the Phagokinetic track migration assay listed in the text $(\mathbf{E}$ and $\mathbf{F})$. For all assays, the same exact number of viable keratinocytes from keloid or surrounding normal skin was initially plated into each well (same for all figures). Data are presented as mean \pm standard deviation (SD). " $p<0.05$ vs. "normal skin" keratinocytes. Bar $=25 \mu \mathrm{m}(\mathrm{E})$. Experiments were repeated three times, and similar results obtained. 
and potent $\mathrm{mTOR}$ kinase inhibitor with an extremely low IC-50 (around $10 \mathrm{nM}$ ) [18, 20]. Studies demonstrate that OSI-027 does not inhibit PI3K and other protein kinases $[18,20]$. In the current study, we showed that OSI-027 disrupted the assembly of mTORC1 (mTOR-Raptor) [14] and mTORC2 (mTOR-Rictor-mLST8) [14, 22] in keloid keratinocytes. Further, OSI-027 blocked phosphorylation of mTORC1 substrates (S6K1, S6 and 4EBP1) as well as the mTORC2 substrate (AKT at Ser-473) [13]. Unlike rapamycin, OSI-027 treatment did not induce the feedback activation of ERK1/2; It blocked p-AKT at Ser-473. Remarkably, OSI-027 demonstrated significantly more
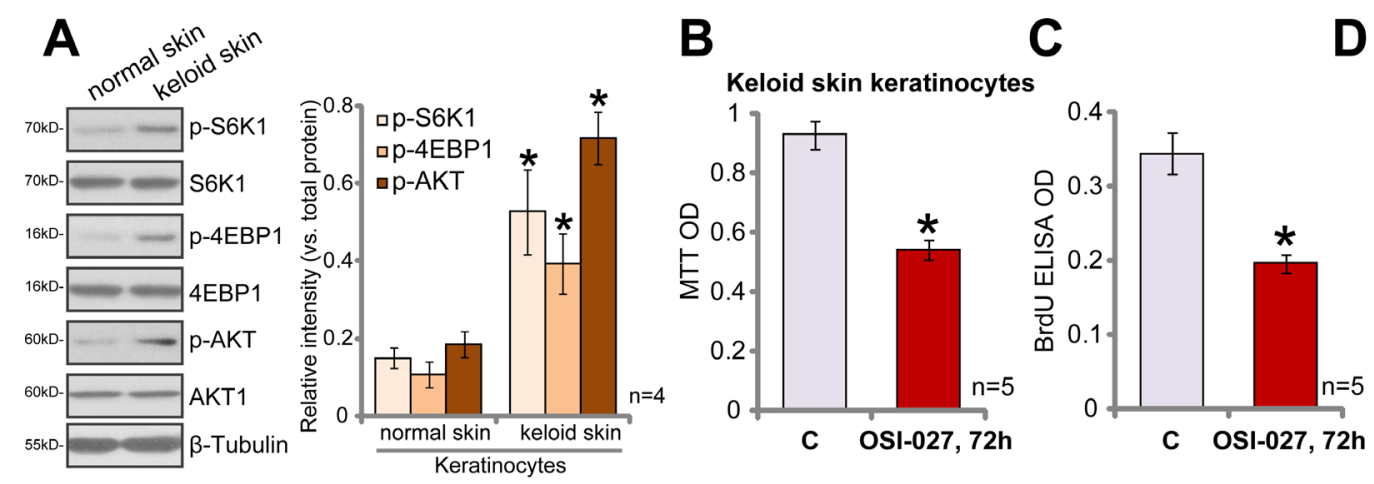

D
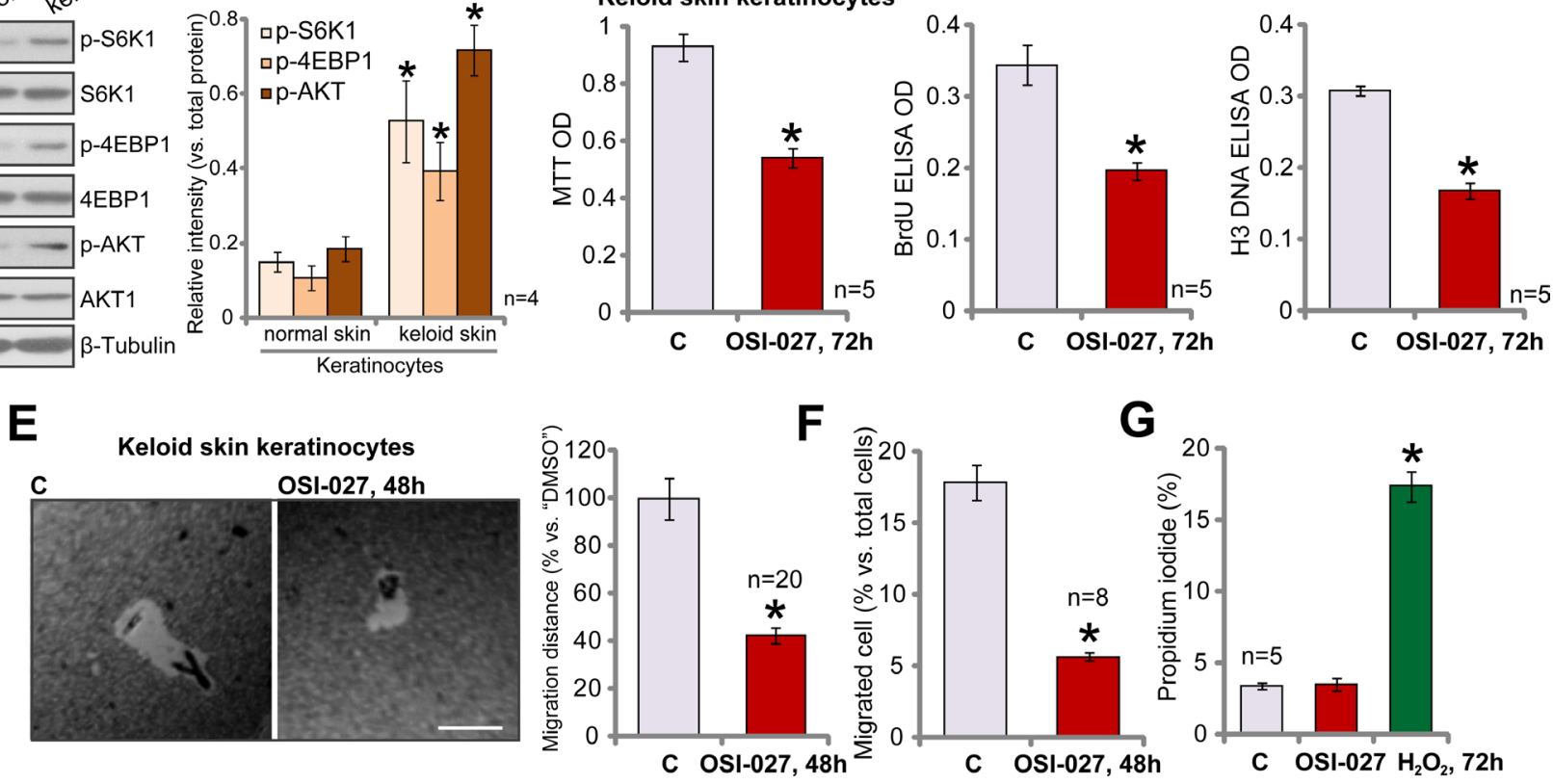

Figure 2: OSI-027 potently inhibits proliferation and migration of keloid keratinocytes. Primary cultured human keratinocytes, derived from keloid and surrounding normal skin, were subjected to a western blotting assay of listed proteins (A, data of four repeated blots were quantified). Primary keloid keratinocytes were treated with OSI-027 (100 nM), vehicle control (0.1\% of DMSO, "C") or $\mathrm{H}_{2} \mathrm{O}_{2}(300 \mu \mathrm{M})$. Cell proliferation $(\mathbf{B}-\mathbf{D}, 72 \mathrm{~h})$, cell migration $(\mathbf{E}$ and $\mathbf{F}, 48 \mathrm{~h})$ and cell death $(\mathbf{G}, 72 \mathrm{~h})$ were tested by the assays listed in the main text. Data are presented as mean \pm standard deviation (SD). " $p<0.05$ vs. "normal skin" keratinocytes (A) or "C" group $(B-G)$. Bar $=25 \mu \mathrm{m}(\mathrm{E})$. Experiments were repeated three times, and similar results obtained.
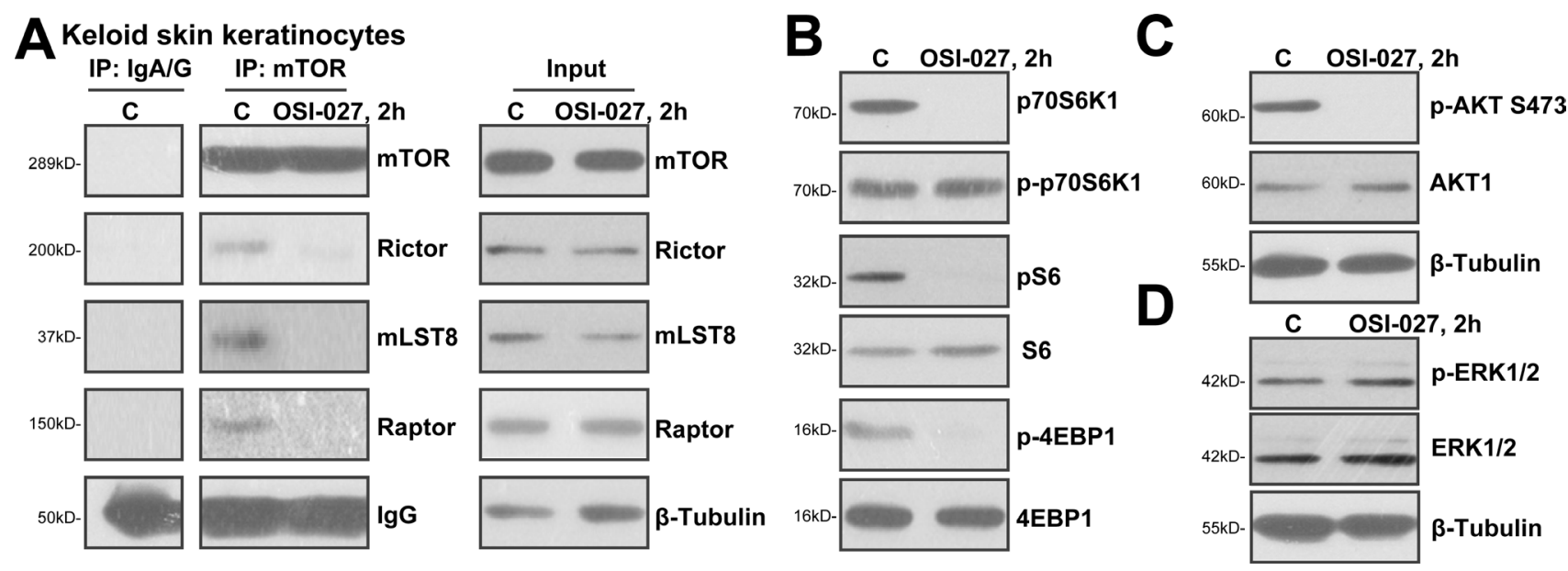

Figure 3: OSI-027 blocks mTORC1 and mTORC2 activation in keloid keratinocytes. Cultured primary keloid keratinocytes were treated with vehicle control (0.1\% of DMSO, "C") or OSI-027 (100 nM) for 2 h. Co-immunoprecipitation (Co-IP) was performed to test the association between mTOR and mTOR complex proteins (Rictor, Raptor and mLST8) (A, left panel). The expression of listed proteins was examined by western blot (A, inputs, and B-D). Experiments were repeated three times, and similar results obtained. 
effective inhibition of the proliferation and migration of keloid keratinocytes compared with rapamycin.

We, here, propose that both mTORC1 and mTORC2 are important in promoting the proliferation and migration of keloid keratinocytes for the following reasons: First, mTORC2, similar to mTORC1, is over-activated in keloid keratinocytes. Second, the mTORC2 substrate AKT (phosphorylation at Ser-473) is known to stimulate cell proliferation and migration $[16,17,32]$. Third, the cocurrent blockage of mTORC1/2 by OSI-027 was more potent than rapamycin (mTORC1 inhibitor) in inhibiting keloid keratinocytes. Additionally, restoring mTORC1 activation by ca-S6K1 only partly attenuated OSI027-induced inhibition of keloid keratinocytes. Lastly,
mTOR2 inhibition, by Rictor siRNAs, inhibited keloid keratinocyte proliferation and migration, less efficiently than OSI-027. Therefore, these in vitro results imply that targeting both mTORC1 and mTORC2 by OSI-027 may have translational value in treating keloid skin.

\section{METHODS}

\section{Chemicals, reagents and antibodies}

OSI-027 (catalog no. S2624) and rapamycin (catalog no. S1039) were obtained from Selleck LLC (Houston, TX, USA). The $\beta$-tubulin antibody (catalog no. T2200) and hydrogen peroxide $\left(\mathrm{H}_{2} \mathrm{O}_{2}\right.$, catalog no. 216763) were
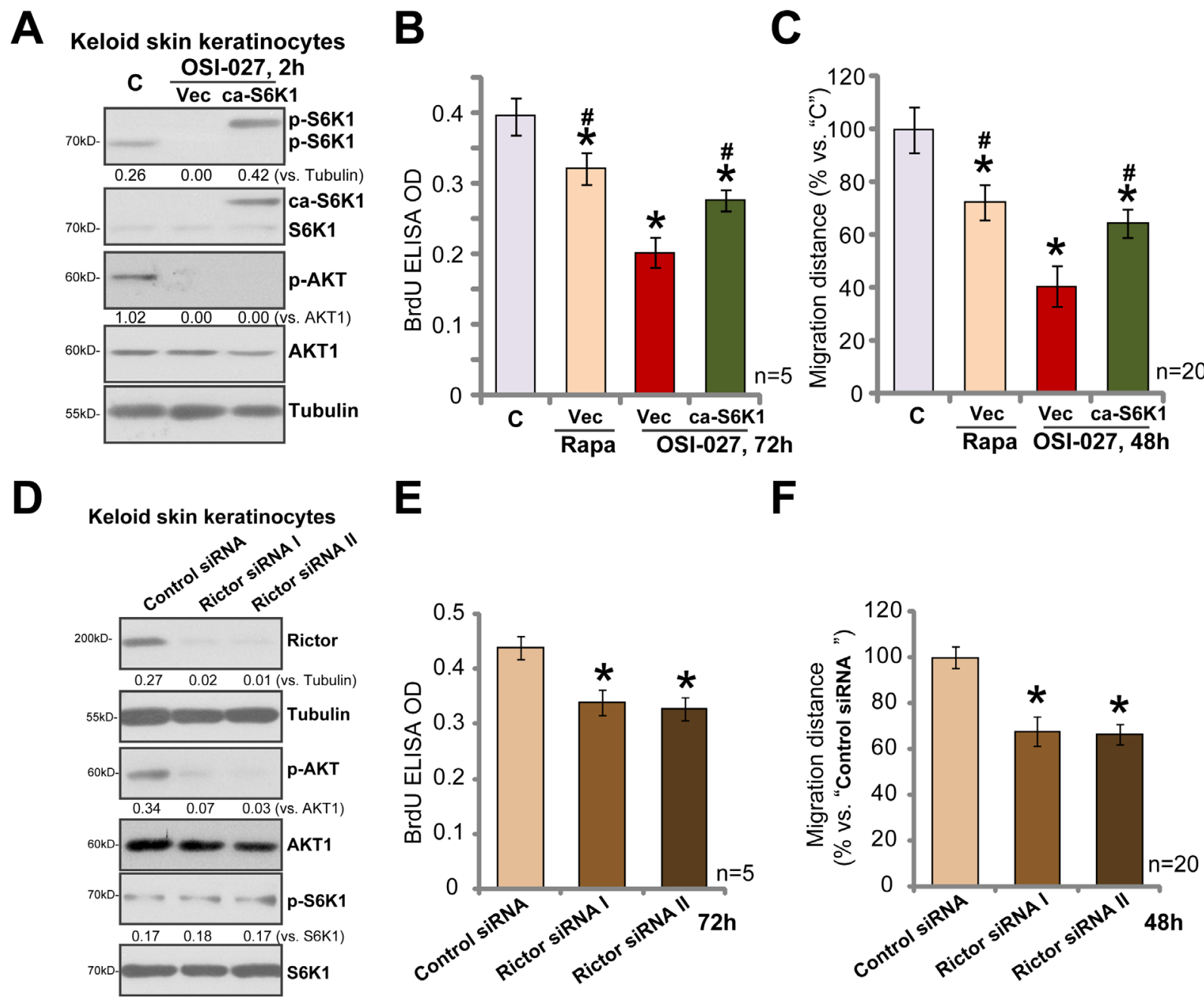

Figure 4: The importance of mTORC1 and mTORC2 in the proliferation and migration of keloid keratinocytes. Stable keloid keratinocytes, expressing empty vector ("Vec", pGCL-flag-puro) or the constitutively active S6K1 (T389E, "ca-S6K1"), were treated with $100 \mathrm{nM}$ of OSI-027 or rapamycin ("Rapa") for the times shown. The expression of listed proteins was examined by western blot (A; data were quantified). Cell proliferation (BrdU ELISA assay; B) and migration (phagokinetic track migration assay; data were quantified in C) were examined. Keloid keratinocytes, transfected with $100 \mathrm{nM}$ of Rictor siRNA ("Rictor siRNA I/II") or scrambled control siRNA ("Control siRNA"), were harvested (after $48 \mathrm{~h}$ of transfection), and the expression of listed proteins is shown (D; data were quantified). Cells were also subjected to BrdU ELISA proliferation (E) and phagokinetic track migration assays (data were quantified in F). Data were presented as mean \pm standard deviation (SD). " $p<0.05$ vs. vehicle control ("C") (B and C); $p<0.05$ vs. OSI-027 treatment in vector cells (B and C). $p<0.05$ vs. "Control siRNA" (E and F). Experiments were repeated three times, and similar results obtained. 
purchased from Sigma-Aldrich (St. Louis, MO, USA). Phosphorylated ("p")-AKT (Ser 473) antibody (9271), AKT1 antibody (2967), p-p70 S6 kinase 1 (S6K, Thr 398) antibody (9209), S6K antibody (9202), p-4E-BP1 (Ser 65) antibody (9451), 4E-BP1 antibody (9644), p-S6 ribosomal protein (S6, Ser 235/236) antibody (2211), S6 antibody (2317), Rictor antibody (2114), Raptor antibody (2280), mLST8 (GßL) antibody (3274), mTOR antibody (2972), p-p44/42 MAPK (ERK1/2) antibody (9102) and ERK1/2 antibody (9102) were all purchased from Cell Signaling Tech.

\section{Culture of primary human keratinocytes}

Epidermal tissues of keloid skin and surrounding normal skin were obtained via biopsy from five informconsent keloid patients (Male, 29/32/35/41/47-year old, administrated at Shanghai Ninth People's Hospital, Shanghai, China). The patients received no prior treatment. The acquired epidermal tissues were minced, washed, and digested by collagenase I (Sigma-Aldrich, catalog no. C0130) [34]. Digestions (2-5) were neutralized, pooled, and filtered. Primary cultures of human keratinocytes were then maintained in Medium 154-CF (Cascade Biologics, M154CF, Portland, OR, USA) supplemented with Human Keratinocyte Growth Supplement plus antibiotics and $\mathrm{Ca}^{2+}$ (catalog no. S0015) [4]. The medium was provided by Dr. Cao [4]. Skin fibroblasts were abandoned. Protocols using human tissues and cells were conducted according to the principles of the Declaration of Helsinki, and were approved by the Ethics Review Board (ERB) of all authors' institutions. Written, informed consents were obtained from the participants.

\section{Cell proliferation assay}

Primary human keratinocytes were seeded into 96 -well tissue culture plates $\left(2 \times 10^{4}\right.$ cells/well $)$. A routine 3-(4,5-dimethyl-thiazol-2-yl)2,5-diphenyl tetrazolium bromide (MTT, Sigma-Aldrich, catalog no. M2128) dye assay was performed to test cell proliferation. The MTT optical density (OD) value at $490 \mathrm{~nm}$ was recorded.

\section{BrdU DNA incorporation assay}

Primary human keratinocytes were seeded into 48 -well tissue culture plates $\left(5 \times 10^{4}\right.$ cells/well $)$ and cultured for $72 \mathrm{~h}$. A 5-bromo-2'-deoxyuridine (BrdU) dye was then added for an additional $16 \mathrm{~h}$. BrdU incorporation into DNA was tested via a BrdU ELISA colorimetric assay (Roche, Shanghai, China, catalog no. 11647229001) according to the manufacturer's protocol. The ELISA OD value at $450 \mathrm{nM}$ was recorded.

\section{$\left[\mathrm{H}^{3}\right]$ Thymidine DNA incorporation assay}

Primary human keratinocytes were seeded into 48well plates $\left(5 \times 10^{4}\right.$ cells/well $)$ and cultured for $72 \mathrm{~h}$ in the presence of $1 \mu \mathrm{Ci} / \mathrm{mL}$ of tritiated thymidine (SigmaAldrich). To determine $\left[\mathrm{H}^{3}\right]$ thymidine incorporation, cells were washed with cold PBS, and DNA precipitated and solubilized as described previously [4]. DNA aliquots were counted and quantified by liquid-scintillation spectrometry, and the $\left[\mathrm{H}^{3}\right]$ thymidine DNA OD recorded.

\section{Phagokinetic track migration assay}

A detailed protocol has been previously described $[21,35,36]$. Briefly, 12-well tissue culture plates were coated with fibronectin $(10 \mu \mathrm{g} / \mathrm{mL}$, Sigma-Aldrich, catalog no. 10838039001), and wells then washed with PBS. Afterwards, a microsphere (Sigma-Aldrich, catalog no. NIST1965) suspension was then added to each well. The plates were then centrifuged at $500 \times g$ at $4{ }^{\circ} \mathrm{C}$ for $20 \mathrm{~min}$. After the supernatant was removed, and freshly trypsinized keratinocytes (1000 cells per well of each treatment) seeded for $48 \mathrm{~h}$, cell migration was observed and photographed under a light microscope.

\section{Transwell assay}

The transwell chamber with $12 \mu \mathrm{m}$ pore filters (Corning, 3403, Shanghai, China) was first pre-coated with $1 \mathrm{mg} / \mathrm{mL}$ Matrigel (BD Biosciences, catalog no. 354230, Suzhou, China). Afterwards, keratinocytes $\left(3 \times 10^{4}\right.$ cells per chamber) in serum-free medium were plated into the upper chamber. Complete medium (with fetal bovine serum [FBS]) was added to the lower chamber. After $48 \mathrm{~h}$ of incubation, cells on the lower surface of the membrane were fixed, stained and then counted in five random fields [37]. Mitomycin ( $1.5 \mu \mathrm{g} / \mathrm{mL}$; Sigma-Aldrich) was always added to exclude the influence of cell proliferation.

\section{Propidium iodide assay for cell death}

After treatment, keratinocytes were washed with icecold PBS, and re-suspended in $70 \%$ alcohol, followed by the addition of DNase-free RNase and propidium iodide (PI, Sigma-Aldrich, catalog no. P4170). The suspension was filtered and subjected to analysis by flow cytometer (FACSCalibur; BD Biosciences). The PI percentage was recorded.

\section{Western blotting assay}

Keratinocytes were lysed via radioimmunoprecipitation assay (RIPA) lysis buffer (Biyuntian, Wuxi, China). Thirty $\mu \mathrm{g}$ of protein lysate per treatment was separated by a SDSpolyacrylamide gel electrophoresis (PAGE) gel, which was then transferred to a polyvinylidene fluoride (PVDF) membrane (Millipore, catalog no. IPVH00010, Shanghai, China). After blocking in 5\% of milk, the blot was incubated with indicated primary and corresponding secondary antibodies. Enhanced chemiluminescence (ECL) reagents (Amersham Biosciences, Shanghai, China) were applied to 
detect antibody-antigen binding. The total gray of each band was quantified via Image J software (NIH, Bethesda, MD). Each lane was loaded with the same amount of quantified protein lysate. The same set of lysate samples were run in sister gels to test different proteins.

\section{Co-immunoprecipitation}

The co-immunoprecipitation (Co-IP) assay protocol has been described previously [38]. Briefly, for each treatment, $800 \mu \mathrm{g}$ protein lysates were pre-cleared with protein A/G beads (Sigma, catalog no. 11719416001) and then incubated with anti-mTOR antibody $(0.2 \mu \mathrm{g})$, overnight. Protein A/G beads ( $30 \mu \mathrm{L}$ per treatment, Sigma) were added afterwards to the lysates again for another $4 \mathrm{~h}$. The beads were then washed with PBS five times, and one time with cell lysis buffer. The integrity of the mTOR complex was then detected by western blotting assay.

\section{Constitutively active S6K1 construct and transfection}

AconstitutivelyactiveS6K1 (T389E, “ca-S6K1-flagpuro") and empty vector (pGCL-flag-puro) were gifts from Dr. Chen [24]. A ca-S6K1 construct was transfected into keratinocytes using Lipofectamine 2000 reagent (Invitrogen, catalog no. 11668027, Shanghai, China). Transfected keratinocytes were then selected by puromycin (2.0 $\mu \mathrm{g} / \mathrm{mL}$, Sigma) for 4 days. The expression of "caS6K1" in stable cells was verified by western blotting assay.

\section{Rictor siRNA}

Keloid keratinocytes were seeded into a six-well tissue culture plate at $1 \times 10^{5}$ cells per well, grown for $24 \mathrm{~h}$, and transfected with Rictor siRNA I (Cell Signaling Technology, \#8649), Rictor siRNA II (Cell Signaling Tech, \#8622), or scramble control siRNA (Cell Signaling Technology, \#6568), at a final concentration of $100 \mathrm{nM}$ using Lipofectamine 2000 reagent (Invitrogen). To exam siRNA efficiency, cells were harvested $48 \mathrm{~h}$ after siRNA transfection, and the silencing of Rictor in transfected cells was confirmed by western blot assay.

\section{Statistics}

Data were expressed as mean \pm standard deviation (SD). The differential significance was calculated using ANOVA (SPSS 16.0). A $p$ value of less than 0.05 was considered statistically significant.

\section{Author contributions}

All authors carried out the experiments, participated in the design of the study and performed the statistical analysis, participated in its design and coordination and helped to draft the manuscript.

\section{CONFLICTS OF INTEREST}

The listed authors have no conflicts of interests.

\section{FUNDING}

This work was supported by a grant from the Fundamental Research Program Funding of Ninth People's Hospital affiliated to Shanghai Jiao Tong University School of Medicine (no. JYZZ009) and National Natural Science Foundation of China (no. 81402618).

\section{REFERENCES}

1. van Leeuwen $\mathrm{MC}$, Bulstra $\mathrm{AE}$, Ket JC, Ritt MJ, van Leeuwen PA, Niessen FB. Intralesional Cryotherapy for the Treatment of Keloid Scars: Evaluating Effectiveness. Plast Reconstr Surg Glob Open. 2015; 3:e437.

2. Jumper N, Paus R, Bayat A. Functional histopathology of keloid disease. Histol Histopathol. 2015; 30:1033-1057.

3. Tuan TL, Nichter LS. The molecular basis of keloid and hypertrophic scar formation. Mol Med Today. 1998; 4:19-24.

4. Zhang YM, Zhang ZQ, Liu YY, Zhou X, Shi XH, Jiang Q, Fan DL, Cao C. Requirement of Galphai1/3-Gab1 signaling complex for keratinocyte growth factor-induced PI3KAKT-mTORC1 activation. J Invest Dermatol. 2015; 135:181-191.

5. Zhang Q, Kelly AP, Wang L, French SW, Tang X, Duong HS, Messadi DV, Le AD. Green tea extract and (-)-epigallocatechin-3-gallate inhibit mast cell-stimulated type I collagen expression in keloid fibroblasts via blocking PI-3K/AkT signaling pathways. J Invest Dermatol. 2006; 126:2607-2613.

6. Syed F, Sherris D, Paus R, Varmeh S, Singh S, Pandolfi PP, Bayat A. Keloid disease can be inhibited by antagonizing excessive mTOR signaling with a novel dual TORC1/2 inhibitor. Am J Pathol. 2012; 181:1642-1658.

7. Syed F, Sanganee HJ, Singh S, Bahl A, Bayat A. Potent dual inhibitors of TORC1 and TORC2 complexes (KU0063794 and KU-0068650) demonstrate in vitro and ex vivo anti-keloid scar activity. J Invest Dermatol. 2013; 133:1340-1350.

8. Khoo YT, Ong CT, Mukhopadhyay A, Han HC, Do DV, Lim IJ, Phan TT. Upregulation of secretory connective tissue growth factor (CTGF) in keratinocyte-fibroblast coculture contributes to keloid pathogenesis. J Cell Physiol. 2006; 208:336-343.

9. Laplante M, Sabatini DM. mTOR signaling in growth control and disease. Cell. 2012; 149:274-293.

10. Gomez-Pinillos A, Ferrari AC. mTOR signaling pathway and mTOR inhibitors in cancer therapy. Hematol Oncol Clin North Am. 2012; 26:483-505, vii.

11. Dancey J. mTOR signaling and drug development in cancer. Nat Rev Clin Oncol. 2010; 7:209-219. 
12. Sabatini DM. mTOR and cancer: insights into a complex relationship. Nat Rev Cancer. 2006; 6:729-734.

13. Saxton RA, Sabatini DM. mTOR Signaling in Growth, Metabolism, and Disease. Cell. 2017; 168:960-976.

14. Sarbassov DD, Ali SM, Kim DH, Guertin DA, Latek RR, Erdjument-Bromage H, Tempst P, Sabatini DM. Rictor, a novel binding partner of $\mathrm{mTOR}$, defines a rapamycininsensitive and raptor-independent pathway that regulates the cytoskeleton. Curr Biol. 2004; 14:1296-1302.

15. Sarbassov DD, Guertin DA, Ali SM, Sabatini DM. Phosphorylation and regulation of Akt/PKB by the rictormTOR complex. Science. 2005; 307:1098-1101.

16. Li H, Lin J, Wang X, Yao G, Wang L, Zheng H, Yang C, Jia C, Liu A, Bai X. Targeting of mTORC2 prevents cell migration and promotes apoptosis in breast cancer. Breast Cancer Res Treat. 2012; 134:1057-1066.

17. Kim EK, Yun SJ, Ha JM, Kim YW, Jin IH, Yun J, Shin HK, Song SH, Kim JH, Lee JS, Kim CD, Bae SS. Selective activation of Akt1 by mammalian target of rapamycin complex 2 regulates cancer cell migration, invasion, and metastasis. Oncogene. 2011; 30:2954-2963.

18. Bhagwat SV, Gokhale PC, Crew AP, Cooke A, Yao Y, Mantis C, Kahler J, Workman J, Bittner M, Dudkin L, Epstein DM, Gibson NW, Wild R, et al. Preclinical characterization of OSI-027, a potent and selective inhibitor of mTORC1 and mTORC2: distinct from rapamycin. Mol Cancer Ther. 2011; 10:1394-1406.

19. Schenone S, Brullo C, Musumeci F, Radi M, Botta M. ATP-competitive inhibitors of mTOR: an update. Curr Med Chem. 2011; 18:2995-3014.

20. Chen B, Xu M, Zhang H, Xu MZ, Wang XJ, Tang QH, Tang JY. The Antipancreatic Cancer Activity of OSI-027, a Potent and Selective Inhibitor of mTORC1 and mTORC2. DNA Cell Biol. 2015; 34:610-617.

21. Cao C, Sun Y, Healey S, Bi Z, Hu G, Wan S, Kouttab N, Chu W, Wan Y. EGFR-mediated expression of aquaporin-3 is involved in human skin fibroblast migration. Biochem J. 2006; 400:225-234.

22. Ali SM, Sabatini DM. Structure of S6 kinase 1 determines whether raptor-mTOR or rictor-mTOR phosphorylates its hydrophobic motif site. J Biol Chem. 2005; 280:19445-19448.

23. Guertin DA, Stevens DM, Thoreen CC, Burds AA, Kalaany NY, Moffat J, Brown M, Fitzgerald KJ, Sabatini DM. Ablation in mice of the mTORC components raptor, rictor, or mLST8 reveals that $\mathrm{mTORC} 2$ is required for signaling to Akt-FOXO and PKCalpha, but not S6K1. Dev Cell. 2006; 11:859-871.

24. Xin Y, Shen XD, Cheng L, Hong DF, Chen B. Perifosine inhibits S6K1-Gli1 signaling and enhances gemcitabineinduced anti-pancreatic cancer efficiency. Cancer Chemother Pharmacol. 2014; 73:711-719.

25. Yu K, Toral-Barza L. Biochemical and pharmacological inhibition of mTOR by rapamycin and an ATP-competitive mTOR inhibitor. Methods Mol Biol. 2012; 821:15-28.
26. Zhou HY, Huang SL. Current development of the second generation of mTOR inhibitors as anticancer agents. Chin J Cancer. 2012; 31:8-18.

27. Zaytseva YY, Valentino JD, Gulhati P, Evers BM. mTOR inhibitors in cancer therapy. Cancer Lett. 2012; 319:1-7.

28. Vilar E, Perez-Garcia J, Tabernero J. Pushing the envelope in the mTOR pathway: the second generation of inhibitors. Mol Cancer Ther. 2011; 10:395-403.

29. Shi Y, Yan H, Frost P, Gera J, Lichtenstein A. Mammalian target of rapamycin inhibitors activate the AKT kinase in multiple myeloma cells by up-regulating the insulinlike growth factor receptor/insulin receptor substrate-1/ phosphatidylinositol 3-kinase cascade. Mol Cancer Ther. 2005; 4:1533-1540.

30. Sun SY, Rosenberg LM, Wang X, Zhou Z, Yue P, Fu H, Khuri FR. Activation of Akt and eIF4E survival pathways by rapamycin-mediated mammalian target of rapamycin inhibition. Cancer Res. 2005; 65:7052-7058.

31. Chen XG, Liu F, Song XF, Wang ZH, Dong ZQ, Hu ZQ, Lan RZ, Guan W, Zhou TG, Xu XM, Lei H, Ye ZQ, Peng EJ, et al. Rapamycin regulates Akt and ERK phosphorylation through mTORC1 and mTORC2 signaling pathways. Mol Carcinog. 2010; 49:603-610.

32. Sparks CA, Guertin DA. Targeting mTOR: prospects for mTOR complex 2 inhibitors in cancer therapy. Oncogene. 2010; 29:3733-3744.

33. Rodon J, Dienstmann R, Serra V, Tabernero J. Development of PI3K inhibitors: lessons learned from early clinical trials. Nat Rev Clin Oncol. 2013; 10:143-153.

34. Phan TT, Lim IJ, Bay BH, Qi R, Longaker MT, Lee ST, Huynh H. Role of IGF system of mitogens in the induction of fibroblast proliferation by keloid-derived keratinocytes in vitro. Am J Physiol Cell Physiol. 2003; 284:C860-869.

35. Ji C, Cao C, Lu S, Kivlin R, Amaral A, Kouttab N, Yang H, Chu W, Bi Z, Di W, Wan Y. Curcumin attenuates EGFinduced AQP3 up-regulation and cell migration in human ovarian cancer cells. Cancer Chemother Pharmacol. 2008; 62:857-865.

36. Cao C, Healey S, Amaral A, Lee-Couture A, Wan S, Kouttab N, Chu W, Wan Y. ATP-sensitive potassium channel: a novel target for protection against UV-induced human skin cell damage. J Cell Physiol. 2007; 212:252-263.

37. Lv Y, Si M, Chen N, Li Y, Ma X, Yang H, Zhang L, Zhu H, Xu GY, Wu GP, Cao C. TBX2 over-expression promotes nasopharyngeal cancer cell proliferation and invasion. Oncotarget. 2017; 8:52699-707. https://doi.org/10.18632/ oncotarget.17084.

38. Zhang Q, Yan HB, Wang J, Cui SJ, Wang XQ, Jiang YH, Feng L, Yang PY, Liu F. Chromatin remodeling gene AT-rich interactive domain-containing protein 1A suppresses gastric cancer cell proliferation by targeting PIK3CA and PDK1. Oncotarget. 2016; 7:46127-41. https://doi.org/10.18632/onco target.10060. 